\title{
Comparative evaluations of the Monte Carlo-based light propagation simulation packages for optical imaging
}

\author{
Lin Wang*, Shenghan Ren ${ }^{\dagger}$ and Xueli Chen ${ }^{\dagger *}$ \\ *School of Information Sciences and Technology \\ Northwest University, Xi'an Shaanxi 710127, P. R. China \\ ${ }^{\dagger}$ Engineering Research Center of Molecular and \\ Neuro Imaging of Ministry of Education \\ School of Life Science and Technology \\ Xidian University, Xi'an Shaanxi 710126, P. R. China \\ txlchen@xidian.edu.cn
}

Received 4 January 2017

Accepted 3 May 2017

Published 9 June 2017

\begin{abstract}
Monte Carlo simulation of light propagation in turbid medium has been studied for years. A number of software packages have been developed to handle with such issue. However, it is hard to compare these simulation packages, especially for tissues with complex heterogeneous structures. Here, we first designed a group of mesh datasets generated by Iso2Mesh software, and used them to cross-validate the accuracy and to evaluate the performance of four Monte Carlobased simulation packages, including Monte Carlo model of steady-state light transport in multilayered tissues (MCML), tetrahedron-based inhomogeneous Monte Carlo optical simulator (TIMOS), Molecular Optical Simulation Environment (MOSE), and Mesh-based Monte Carlo (MMC). The performance of each package was evaluated based on the designed mesh datasets. The merits and demerits of each package were also discussed. Comparative results showed that the TIMOS package provided the best performance, which proved to be a reliable, efficient, and stable MC simulation package for users.
\end{abstract}

Keywords: Light transport; Monte Carlo; comparative evaluation; mesh datasets.

\section{Introduction}

Optical imaging techniques play an important role in preclinical researches, such as cancer detection and drug development. ${ }^{1,2}$ The study of light propagation is essential for optical imaging, especially for threedimensional (3D) optical imaging. The radiative

\$Corresponding author.

This is an Open Access article published by World Scientific Publishing Company. It is distributed under the terms of the Creative Commons Attribution 4.0 (CC-BY) License. Further distribution of this work is permitted, provided the original work is properly cited. 
transfer equations (RTE) can be used for modeling the light propagation in tissues accurately. Many numerical approximations have been proposed to solve the $\mathrm{RTE},{ }^{3,4}$ however, it is still a challenging task to effectively solve it in heterogeneous tissues. Monte Carlo (MC) method has been commonly used for the simulation of light propagation in tissues by simulating a large number of photon trajectories. The physical quantities, such as the photon absorbance, reflectance, and transmittance are usually recorded. Because it is a statistics-based method, the MC simulation result is often used as the gold standard to validate other numerical solutions.

The MC simulation of light propagation has been developed rapidly over the past decades. Many simulation packages were also consequently developed. Based on how the tissues or phantom are represented, the MC simulation packages can be divided into five categories as follows: the multilayered, voxel-based, surface-based, tetrahedronbased, and mesh-based packages. Wang et al. developed a Monte Carlo model of steady-state light transport in multi-layered tissues (MCML), ${ }^{5}$ in which the phantom is represented by regularly geometrical shapes. MCML has been widely used for MC simulation and becomes a benchmark for the simulation of photon propagation in tissues, but it can only cope with layered infinite media. Recently, several improved versions of MCML were also developed to handle with the multilayered medium that was embedded with spherical, cylindrical, ellipsoidal, or cuboidal objects. ${ }^{6-8}$ In order to deal with 3D complex, heterogeneous structures, Boas et al. developed voxel-based MC method, called tMCimg. ${ }^{9}$ Furthermore, Margallo-Balbas et al. ${ }^{10}$ and Ren et $a .^{11,12}$ developed surface-based MC methods. In these methods, the triangular meshes, a kind of data sets, were used to depict complex surface or boundary of the tissues or organs accurately. However, due to the large number of triangular meshes at boundary, it is difficult to determine the position of the photon relative to the triangular meshes. The calculation of photon-triangle intersection requires a heavy computation. Shen et al. developed a tetrahedron-based inhomogeneous MC optical simulation (TIMOS) method. ${ }^{13}$ The advantage of TIMOS lies in its efficiency to determine photon-triangle intersection. This is because only four photon-triangle intersections need to be considered when a photon travels in the tetrahedron. Meanwhile, Fang et al. proposed a mesh-based
Monte Carlo (MMC) method which uses a generalized ray-tracing model to calculate photon-triangle intersection. ${ }^{14}$ In addition, other MC methods were also developed for specific applications, such as laser irradiation or interaction with skin tissue and brain studies. ${ }^{15-22}$

On the other hand, the phantom models of MC simulation tools vary significantly. The formats of the recorded results are also different. The arising problem is how to compare these packages accurately and to assess them fairly. Several studies have investigated such a problem. TIMOS was compared with MCML and the Molecular Optical Simulation Environment (MOSE, a surface-based MC simulation package) in multi-layered and complex geometry, respectively. ${ }^{13}$ The comparisons were conducted in terms of both the accuracy and efficiency. TIMOS was also compared with MMC and the CUDA accelerated MCML ${ }^{23}$ in terms of the absorbed fraction and simulation time. The pseudocode of the TIMOS and the MMC scheme in Plücker coordinate were also compared. ${ }^{24}$ In spite of these efforts, there is still a need to build a standard dataset to compare these MC simulation packages. The standard dataset can also be used to evaluate the newly developed MC packages and numerical solutions.

Here, we presented a dataset of three phantoms that are generated by Iso2Mesh. ${ }^{25}$ Four MC-based simulation packages, including MCML, MOSE, TIMOS and MMC, are compared and cross-validated using the dataset. This paper is organized as follows. The principle of the MC simulation of the light propagation, the MC-based simulation packages, and the designed dataset are introduced in the next section. The simulation results are compared and discussed in the end.

\section{Methods and Materials}

\subsection{Monte Carlo method for light propagation}

The MC method for light propagation is based on randomly recording a large number of photons trajectories in tissues. The method is statistics in nature and based on random sampling of variables. The step size of single photon and the deflection angle between each two steps are statistically calculated according to the optical properties of the tissues, including the absorption coefficient, the 
scattering coefficient, the reflective index, and the anisotropy factor. Although the surface, tetrahedron, and mesh-based models can depict the tissue boundary more accurately than multi-layered or voxel-based models, the descriptions of light propagation inside the tissues for all the models are the same. The flow chart of the MC simulation of light propagation in multi-layered tissue is shown in Fig. 1.

The photons are generated according to the settings of the light source, such as the light beam directly emitting into the tissues, point or regularshaped light source that can be represented by regularly geometrical shapes inside the tissues. The settings of the light source can be classified according to the modalities of optical imaging, such as the bioluminescence imaging (BLI), fluorescence molecular imaging (FMI), diffuse optical imaging (DOI), etc. The light source of BLI is initialized

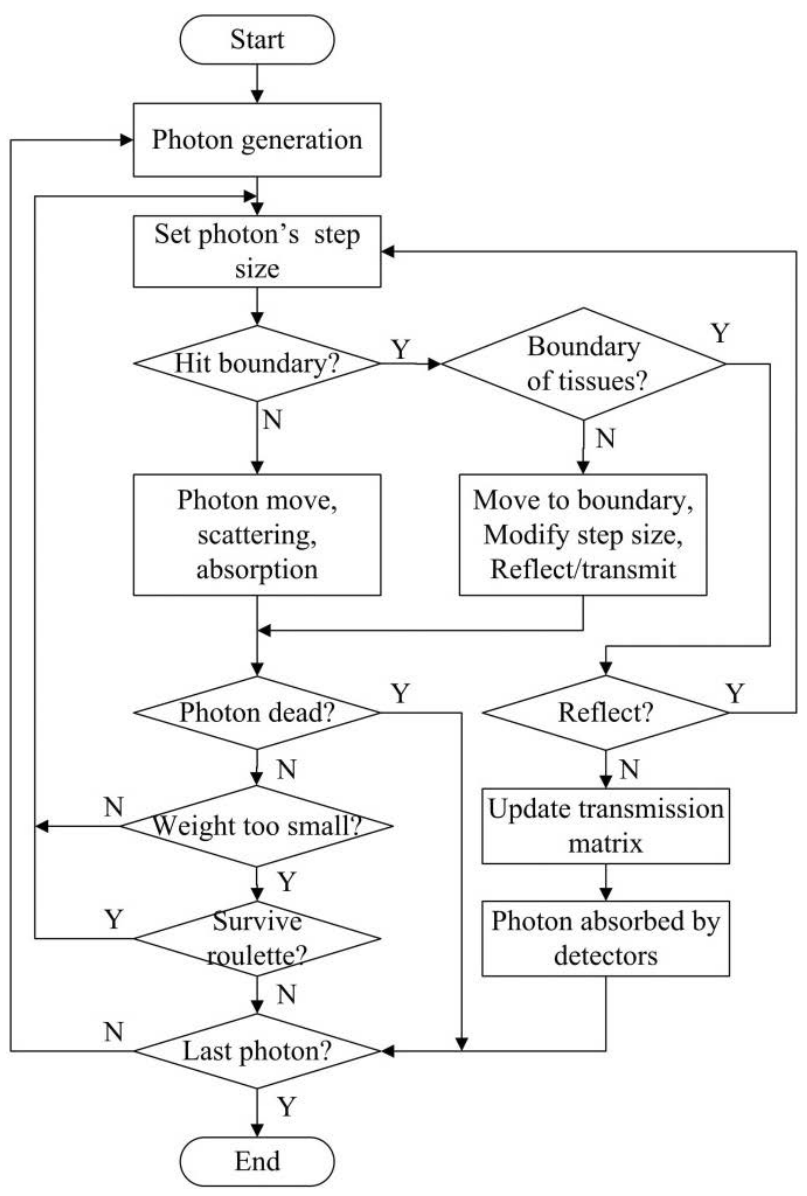

Fig. 1. Flow chart of MC simulation of light propagation in tissues. Here, "Boundary of tissues" means the boundary between the tissue and ambient medium. inside the tissues. The initial direction of a photon is determined by randomly sampling the space angle. There are two types of light sources in FMI, the excitation and emission light sources. The excitation light source is located outside the phantom and projected into it. The emission light source is determined by the distribution of the fluorophores inside the tissues. The DOI system is similar to FMI system except that the DOI system doesn't contain the emission light source. The detailed description of the MC algorithm can be found in literatures. ${ }^{5,11,12}$

\section{2. $M C M L$}

MCML deals with the problem that a light beam transports in the multilayered tissues. ${ }^{5}$ It is incapable of simulating light propagation in $3 \mathrm{D}$ heterogeneous tissues. The reflectance, absorption, and transmittance are recorded in a cylindrical coordinate system. The light source in MCML can be set as a narrow light beam perpendicular to the layers. The parameters of MC simulation include the optical parameters of each layer, the depth of each layer, the resolution of the phantom, and the photon number of the light source.

\subsection{MOSE}

The simulation of light propagation in MOSE is similar to that of MCML. ${ }^{11,12}$ The major difference is that the multilayered tissue model is replaced by $3 \mathrm{D}$ complex heterogeneous model that can be described by the regularly geometrical shape-based objects or triangular meshes. The triangular meshes are a kind of data sets that can be used to describe the surface of tissues or organs. The light source in MOSE can be set as 3D regular shapes or triangular meshes. The various setting of the light sources makes MOSE capable to simulate nearly all the optical imaging modalities such as BLI, DOI, and FMI. Although the triangular meshes can model the boundary of the tissues accurately, it is time-consuming to determine the position of the photon and the photon-boundary intersection. The position of the intersection must be calculated by searching a large number of triangular meshes. For each tissue whose surface is represented by a number of closed triangular meshes, its optical parameters can only 
be set as unmixed which is inaccurate for real applications.

\subsection{TIMOS}

TIMOS utilizes tetrahedral meshes as the simulated model. ${ }^{13}$ The phantom used in photon propagation is discretized into tetrahedral meshes, which are numbered in a list and the neighborhoods of all the meshes are calculated before running the simulation. It is easy to determine the position of the photon because it can only be in a certain tetrahedron. The advantage of the TIMOS over MOSE is that there are only four triangles in each tetrahedron, which means that it only needs four calculations of the ray-triangle intersection to determine the photon-boundary intersection. The setting of the optical parameters for TIMOS is more flexible than that of MOSE because each tetrahedron can represent a tissue in TIMOS while a list of triangular meshes is needed in MOSE.

\section{5. $M M C$}

MMC package is similar to TIMOS. ${ }^{14}$ The major difference between them is that MMC uses a generalized ray-tracing algorithm and the Plücker coordinate system. The advantage of MMC is that it can handle not only the tetrahedron but also other polyhedrons. MMC also supports MC simulation of light propagation in time domain and frequency domain. ${ }^{26,27}$
A general review of these MC simulation packages is summarized in Table 1.

\subsection{Dataset description}

We designed three simulation phantoms in the dataset for MC simulation. The phantoms are different in structures from simple to complex, including multi-layered tissues, cube-shaped 3D phantom, and digital mouse. The meshes of the dataset were generated by Iso2Mesh which was developed by Fang et al. ${ }^{25}$

The multi-layered tissues are usually used for MC simulation of DOI system. As shown in Fig. 2(a), the phantom with a radius of $10 \mathrm{~cm}$ and a height of $2 \mathrm{~cm}$ contains five layered slabs. The depths of each layer from top to bottom are $0.1 \mathrm{~cm}$, $0.2 \mathrm{~cm}, 0.2 \mathrm{~cm}, 0.9 \mathrm{~cm}$, and $0.6 \mathrm{~cm}$, respectively. Each layer represents an unmixed tissue. The information of the grid size used here is set as: $\mathrm{dr}=0.02 ; \mathrm{dz}=0.02$; number of $\mathrm{dr}$ is 200 , and that of $\mathrm{dz}$ is 100 . In order to compare MCML with the mesh-based MC packages, the multi-layered phantom is discretized into 666,624 nodes and 3,771 , 482 tetrahedrons.

The second phantom is a regular-shaped phantom with a sphere in a cube, as shown in Fig. 2(b). The cube has a dimension of $6 \times 6 \times 6 \mathrm{~cm}^{3}$. The sphere has a radius of $2 \mathrm{~cm}$ and is located at the center of the phantom with the coordinate of $(0,0,0) \mathrm{cm}$. The phantom contains 35, 991 nodes and 209, 847 tetrahedrons. The mesh size is around $0.1 \mathrm{~cm}$.

Table 1. Summary of MC-based simulation packages for light propagation.

\begin{tabular}{|c|c|c|c|c|}
\hline Packages & Tissue type & $\begin{array}{c}\text { Recorded } \\
\text { transmittance }\end{array}$ & Recorded absorption & Main feature \\
\hline MCML & multilayer & $\begin{array}{l}\text { Cylindrical } \\
\text { coordinate system. }\end{array}$ & $\begin{array}{l}\text { Cylindrical coordinate } \\
\text { system. }\end{array}$ & $\begin{array}{l}\text { Determines the photon's position } \\
\text { and trajectory according to the } \\
\text { spatial position in } 3 \mathrm{D} \\
\text { coordinate system. }\end{array}$ \\
\hline MOSE & Triangular meshes & $\begin{array}{l}\text { Boundary surface } \\
\text { and nodes. }\end{array}$ & $\begin{array}{l}\text { Voxels inside the triangular } \\
\text { meshes in Cartesian } \\
\text { coordinate system. }\end{array}$ & $\begin{array}{l}\text { Determines the photon's position } \\
\text { by searching a list of triangular } \\
\text { meshes. }\end{array}$ \\
\hline TIMOS & Tetrahedral meshes & $\begin{array}{l}\text { Boundary surface } \\
\text { and nodes. }\end{array}$ & Each tetrahedral mesh. & $\begin{array}{l}\text { Easy to track the photon's } \\
\text { position and path inside the } \\
\text { tetrahedrons. }\end{array}$ \\
\hline MMC & Tetrahedral meshes & Boundary nodes. & Each node or each element. & $\begin{array}{l}\text { Same as TIMOS but determines } \\
\text { the photon-triangle intersection } \\
\text { in a Plucker coordinate. }\end{array}$ \\
\hline
\end{tabular}




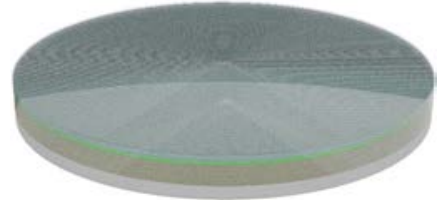

(a)

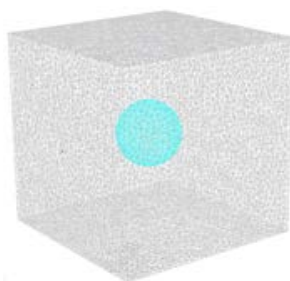

(b)

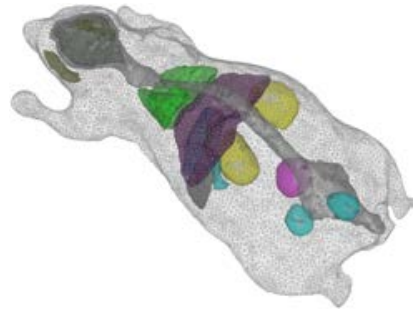

(c)

Fig. 2. Structure of the phantoms for the comparisons of MC-based simulation packages. (a) Multilayered tissues, (b) Regularly shaped tissue, and (c) Digital mouse.

The third phantom is a digital mouse model generated from the Digimouse atlas, ${ }^{28}$ containing 17 different tissues as shown in Fig. 2(c). The phantom consists of 153,015 nodes and 853,949 tetrahedral elements. The range of the mouse is located in the region of interest of $\{(x, y, z) \mid 0.256<x<3.765$, $0.141<y<7.8,0.115<z<2.045\} \mathrm{cm}$.

\section{Experiments and Results}

The first experiment is conducted to compare the four MC packages by using multi-layered tissues. The light source is projected into the multi-layered tissues on the top center of the phantom. The optical parameters of the tissues and the refractive index of the environment are shown in Table 2. Each simulation runs with $10,000,000$ photon packets. The distributions of the diffuse reflectance and transmittance obtained by MOSE are presented in Figs. 3(a) and 3(b), respectively. Figure 4(a) shows the comparison of the transmittance between MCML and MOSE as the radial distance increases, and Fig. 4(b) shows the comparison of the transmittance between TIMOS and MOSE at different triangular meshes. Here, MOSE is operated at the cylindrical coordinate (the regularly geometrical structure) for Fig. 4(a) and tetrahedron for Fig. 4(b). From Fig. 4, a good agreement is obtained between MCML, MOSE and TIMOS, which demonstrated the consistency of these MC simulation packages. The normalized root mean square error (NRMSE) is applied to evaluate the discrepancy between them, which is defined as

$$
\bar{e}=\sqrt{\frac{1}{N} \sum_{i=1}^{N}\left(d_{i}^{1}-d_{i}^{2}\right)^{2}},
$$

where $N$ is the dimension of the resulting data, $d$ is the photon flux density at nodes. The NRMSE between MOSE and TIMOS is $0.0388 \%$, and that between MOSE and MCML is $0.06 \%$. The result shows a good agreement between MOSE, MCML and TIMOS.

We didn't compare with MMC because it can only handle the problem when the refractive indices of the layers and the environment are the same. We changed the refractive indices of all the layers and of the environment to 1.4 and used absorbed fractions to compare all the $\mathrm{MC}$ packages. The absorbed fractions of MCML, MOSE, TIMOS, and MMC are $0.5711,0.5684,0.5709$ and 0.5712 , respectively. The simulation times of MCML, MOSE, TIMOS, and MMC are $754,886,377.1$ and 452 sec, respectively

Table 2. Optical properties of the multilayered tissues.

\begin{tabular}{lccccc}
\hline Layer index & Depth $(\mathrm{cm})$ & Absorption coefficient $\left(\mathrm{mm}^{-1}\right)$ & $\begin{array}{c}\text { Scattering } \\
\text { coefficient }\left(\mathrm{mm}^{-1}\right)\end{array}$ & Anisotropic factor & Reflective index \\
\hline 1 & 0.1 & 0.43 & 10.7 & 0.79 & 1.5 \\
2 & 0.2 & 0.27 & 18.7 & 0.82 & 1.4 \\
3 & 0.2 & 0.33 & 19.2 & 0.82 & 1.4 \\
4 & 0.9 & 0.27 & 18.7 & 0.82 & 1.4 \\
5 & 0.6 & 0.34 & 19.4 & 0.82 & 1.4 \\
\hline
\end{tabular}




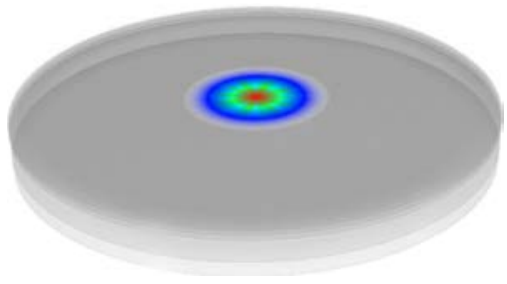

(a)

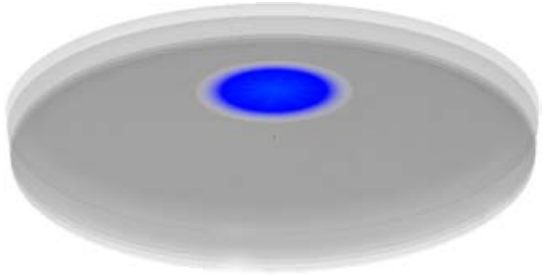

(b)

Fig. 3. Simulation results of the MOSE in the multilayered phantom. (a) Diffuse reflectance and (b) Transmittance.

on Ubuntu 12.04 with 8 GB RAM and Intel Xeon Processor E5620 (2.4 GHz). The following experiments are conducted in the same environment. The result shows that the absorbed fractions of these
MC packages are almost the same while TIMOS have a better efficiency over others.

The second experiment uses the regular-shaped phantom. A point light source is incident onto one

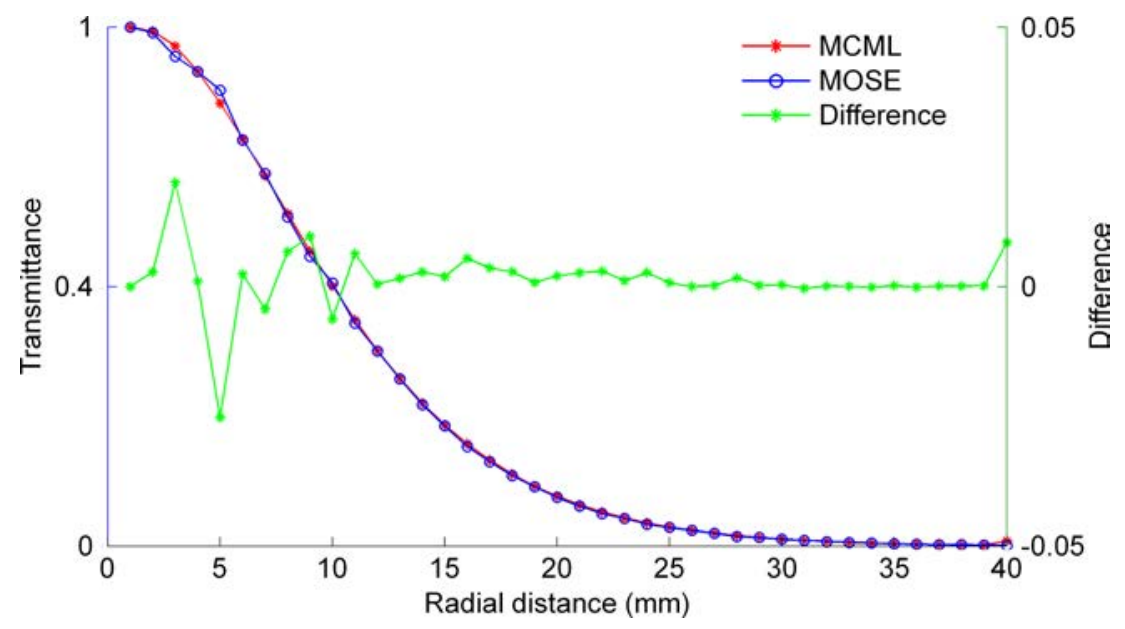

(a)

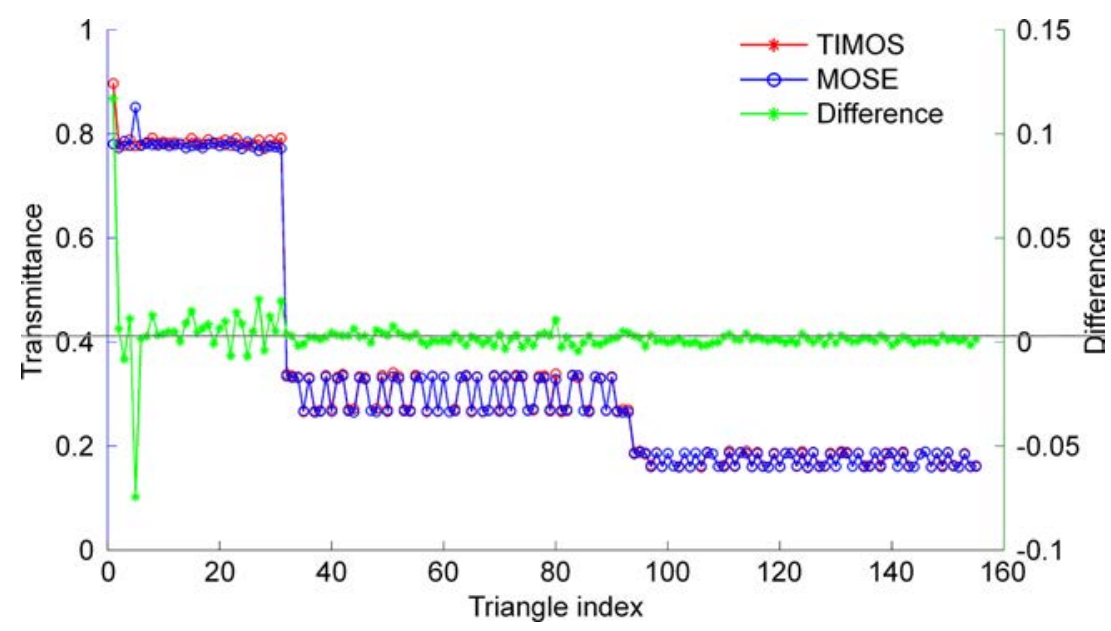

(b)

Fig. 4. Comparisons of the transmittance between the MCML, TIMOS, and MOSE in case of multilayered phantom. (a) Comparison between the MCML and MOSE at different radial distance. (b) Comparison between the TIMOS and MOSE at different triangle. 
Table 3. Optical properties of the regular-shaped phantom

\begin{tabular}{lcccc}
\hline Tissues & Absorption coefficient $\left(\mathrm{mm}^{-1}\right)$ & Scattering coefficient $\left(\mathrm{mm}^{-1}\right)$ & Anisotropic factor & Reflective index \\
\hline Cube & 0.002 & 1 & 0.01 & 1.37 \\
Sphere & 0.05 & 5 & 0.9 & 1.37 \\
\hline
\end{tabular}

of the side surfaces of the cube with the coordinate of $(0,-0.3,0) \mathrm{cm}$. The optical parameters of the phantom are shown in Table 3. The classical MCML can only handle the multilayered tissues, so MOSE and TIMOS are compared in this experiment. The transmittance result of MOSE is shown in Fig. 5(a). The comparison of the transmittance between TIMOS and MOSE at different triangle meshes is shown in Fig. 5(b). The NRMSE between TIMOS and MOSE is $0.00346 \%$. Also, we change the refractive indices to the same for the tissues and environment and conduct the comparison among the MC packages of MOSE, TIMOS, and MMC. The absorbed fractions of MOSE, TIMOS, and MMC are 0.1098, 0.1097, and 0.1096, respectively. The simulation time of MOSE, TIMOS, and MMC is 1238,261 , and $620 \mathrm{sec}$, respectively. To investigate the dependence of the simulation time on mesh size, we change the number of triangular or tetrahedral meshes and record the simulation time of MOSE and TIMOS (Fig. 6). Results show that the simulation time is of positive correlation to the

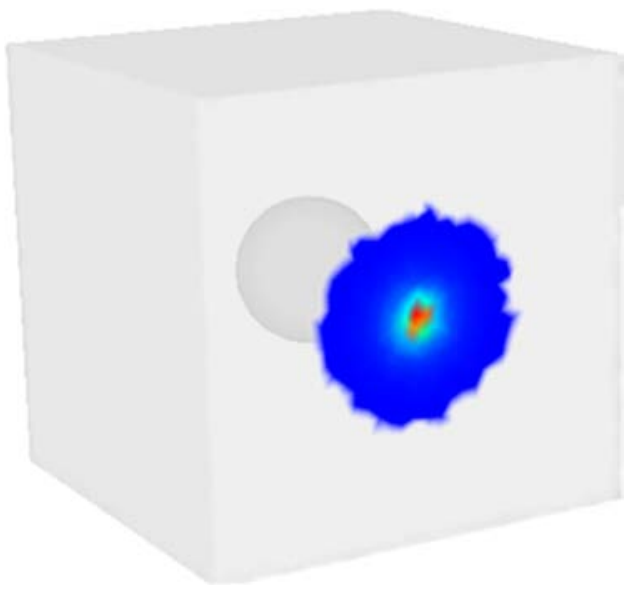

(a)

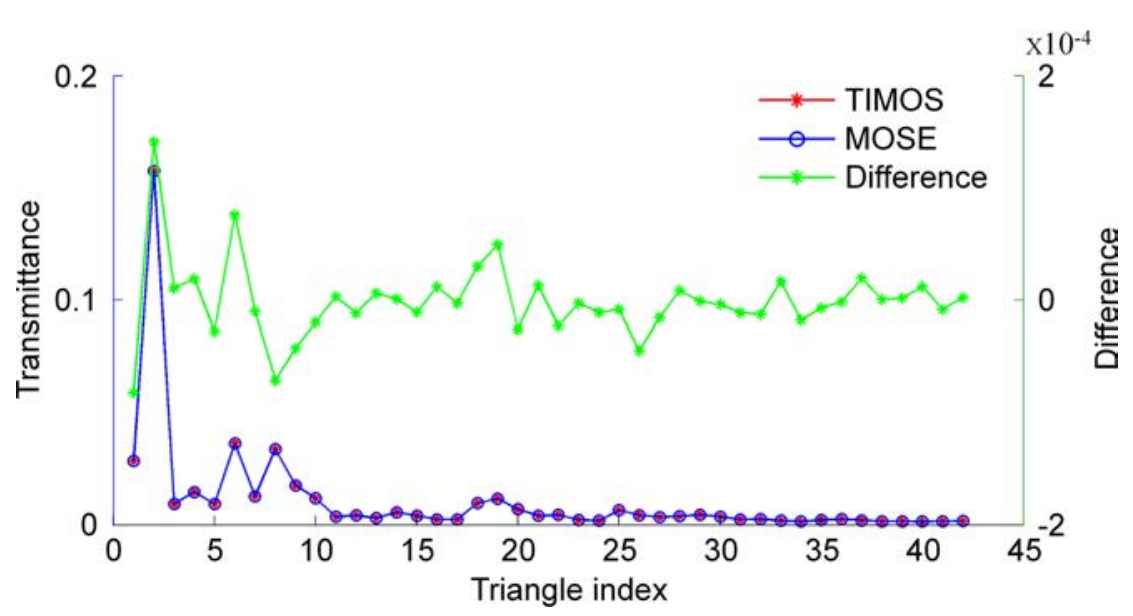

(b)

Fig. 5. Comparisons of the transmittance between the TIMOS and MOSE in case of regular-shaped phantom. (a) The transmittance result of MOSE. (b) Comparison of the transmittance between TIMOS and MOSE. 


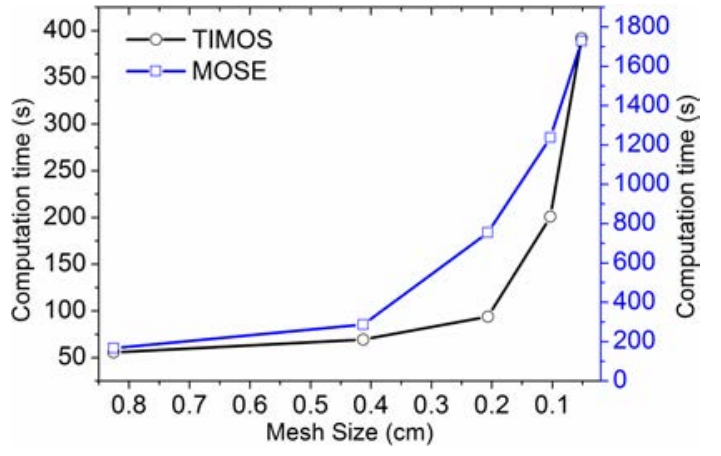

Fig. 6. Simulation time of TIMOS and MOSE as a function of mesh size.

variations in mesh or tetrahedron density. Especially, the more dense the mesh is, the more obvious the advantage of TIMOS in simulation time is. The results collectively show that these MC packages have a good consistency while TIMOS runs faster than others in the investigated case.

The third experiment is conducted using the digital mouse phantom. The light source is illuminated on the back of the mouse with the coordinate of $(1.5,4.2,1.2) \mathrm{cm}$. The emission wavelength of the light source is $620 \mathrm{~nm}$. The digital mouse contains 17 different organs. The optical properties of the organs are calculated according to Ref. 29. The transmittance result of MOSE is illustrated in Fig. 7(a). The comparison between TIMOS and MOSE at different triangular meshes is shown in Fig. 7(b). The NRMSE between TIMOS and MOSE is 0.0647 . MMC records the transmittance on the nodes of the surface, so we again compare the absorbed fractions instead. The absorbed fractions of MOSE, TIMOS and MMC are 0.9997, 0.9997, and 0.9994 , respectively, showing a good consistency. The simulation time for MOSE, TIMOS, and MMC are 3032, 1086 and $1305 \mathrm{sec}$, respectively.

\section{Discussions and Conclusion}

The MC simulation of light propagation in tissues is essential for the study of three-dimensional optical imaging. As the golden standard for verifying other numerical methods, some MC-based simulation packages have been rapidly developed. However, there is no standard to determine the accuracy and performance of these MC simulation packages. Here, we construct a dataset that includes a multilayered tissue, cube-shaped heterogeneous phantom and digital mouse-based phantom, to cross-validate and evaluate the performance of four MC simulation packages. The evaluated simulation packages include MCML, MOSE, TIMOS, and MMC. From the comparative results, two phenomena could be observed. First, a good consistency for simulated transmittance or reflectance is achieved among four simulation packages. This is because the core algorithms that describe the behaviors of photons in tissues are the same for all the simulation packages. All of them are originated from the classical MCML.

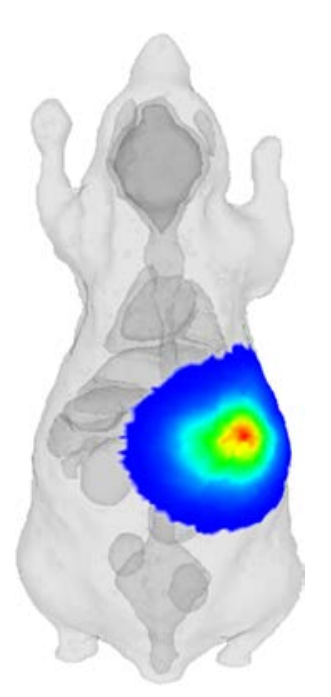

(a)

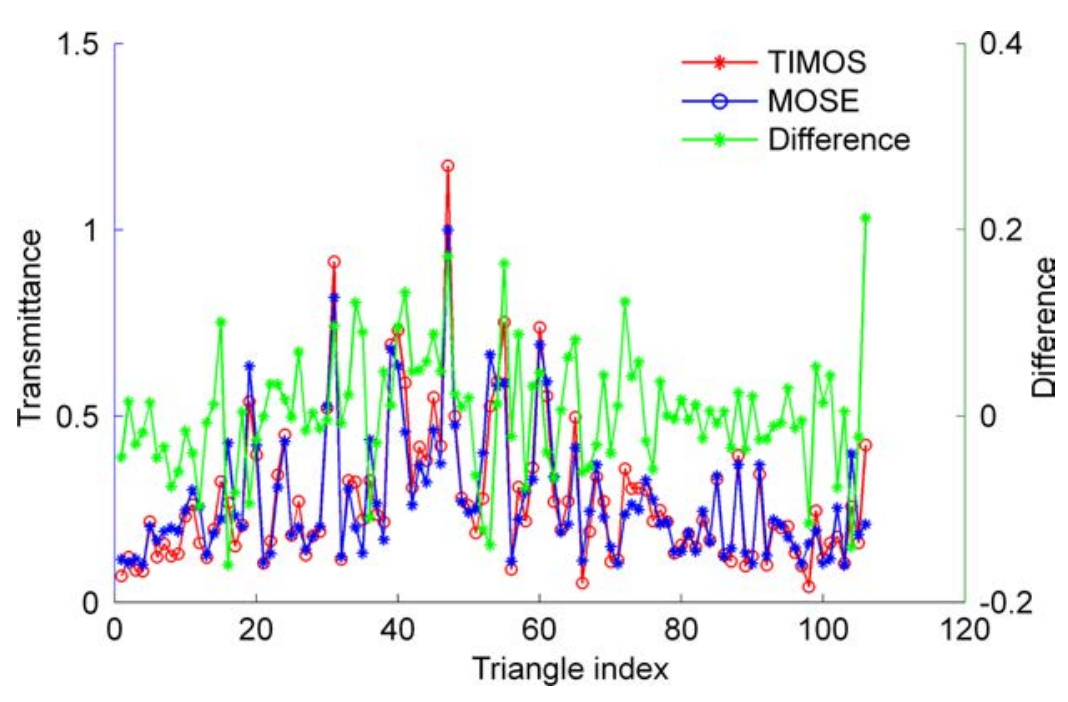

(b)

Fig. 7. Comparisons of the transmittance between the TIMOS and MOSE in case of digital mouse phantom. (a) The transmittance map of MOSE. (b) Comparison of the transmittance between TIMOS and MOSE at different triangular meshes. 
Second, the simulation efficiency is different for four simulation packages. This should be caused by the different representation of the tissues. In our designed dataset, the tetrahedron- or mesh-based methods (TIMOS or MMC) provide the better efficiency than others. Furthermore, TIMOS is a little faster than MMC. Compared with MOSE in which the position of photon-boundary intersection must be calculated by searching a large number of triangular meshes, it is much easier for TIMOS to do this because there are only four triangular meshes in each tetrahedron. Thus, TIMOS is always much faster than MOSE no matter how dense the mesh is (as shown in Fig. 6). Compared with MMC which runs the calculation in Plücker coordinate system, the TIMOS that operates in Cartesian coordinate system is a bit more efficient. Moreover, MCML is a single-thread-based package, but TIMOS can be operated in multi threads. All in all, TIMOS should prove to be a reliable and efficient $\mathrm{MC}$ simulation package for applications.

On the other hand, other simulation packages have their own merits as well. The classical MCML can only handle with multi-layered tissues, but it is the foundation of other packages and can be easy to modify. MOSE may need a little more time to run simulation, but it is user-friendly and can be easy to grasp by non-professional users. TIMOS and MMC provide better performance in terms of efficiency; however, they need to write a script program to run a simulation which may need some professional operations.

The main drawback of the MC simulation is the heavy burden of computation. In order to solve this problem, some GPU accelerated packages are also developed, such as GPU-MOSE, ${ }^{11}$ GPU-MCML ${ }^{30}$ and MCX, ${ }^{31}$ etc. Compared with the CPU-based acceleration strategy, GPU-based parallel computation has the advantages of low cost and easy accessibility. Our future work will concentrate on the development and the assessment of GPU-based MC tools. More datasets will be created and prepared for the evaluation of these MC tools.

\section{Acknowledgments}

This work is partly supported by the National Natural Science Foundation of China under Grant Nos. 81571725 and 81230033. We appreciate Dr. Qianqian Fang of Northeastern University,
Dr. Haiou Shen of the Math Works Inc., Prof. Jimin Liang of Xidian University, and Prof. Ge Wang of Rensselaer Polytechnic Institute for helpful discussions.

\section{References}

1. V. Ntziachristos, J. Ripoll, L. V. Wang, R. Weissleder, "Looking and listening to light: The evolution of whole-body photonic imaging," Nat. Biotechnol. 23(3), 313-320 (2005).

2. R. Weissleder, M. Pittet, "Imaging in the era of molecular oncology," Nature 452(7187), 580-589 (2008).

3. K. Liu, Y. Lu, J. Tian et al., "Evaluation of the simplified spherical harmonics approximation in bioluminescence tomography through heterogeneous mouse models," Opt. Express 18(20), 20988$21002(2010)$.

4. X. He, H. Guo, J. Yu, X. Zhang, Y. Hou "Effective and robust approach for fluorescence molecular tomography based on CoSaMP and SP3 model," J. Innov. Opt. Heal. Sci. 9(6), 1650024 (2016).

5. L. Wang, S. Jacques, L. Zheng, "MCML - Monte Carlo modeling of light transport in multi-layered tissues," Comput. Methods Programs Biomed. 47(2), 131-146 (1995).

6. V. Periyasamy, M. Pramanik, "Monte Carlo simulation of light transport in turbid medium with embedded object-spherical, cylindrical, ellipsoidal, or cuboidal objects embedded within multilayered tissues," J. Biomed. Opt. 19(4), 045003 (2014).

7. V. Periyasamy, S. Sanchita, D. Gagan, A. Freek, S. Umapathy M. Pramanik, "Experimentally validated Raman Monte Carlo simulation for a cuboid object to obtain Raman spectroscopic signatures for hidden material," J. Raman Spectrosc. 46(7), 669-676 (2015).

8. K. Sivasubramanian, V. Periyasamy, K. Wen, M. Pramanik, "Optimizing light delivery through fiber bundle in photoacoustic imaging with clincial ultrasound system: Monte Carlo simulation and experimental validation," J. Biomed. Opt. 22(4), 041008 (2016).

9. D. Boas, J. Culver, J. Stott, A. Dunn, "Three dimensional Monte Carlo code for photon migration through complex heterogeneous media including the adult human head," Opt. Express 10(3), 159-170 (2002).

10. E. Margallo-Balbas, P. French, "Shape based Monte Carlo code for light transport in complex heterogeneous tissues," Opt. Express, 15(21), 1408614098 (2007).

11. N. Ren, J. Liang, X. Qu, J. Li, B. Lu, J. Tian, "GPU-based Monte Carlo simulation for light 
propagation in complex heterogeneous tissues," Opt. Express, 18(7), 6811-6823 (2010).

12. S. Ren, X. Chen, H. Wang, X. Qu, G. Wang, J. Liang, J. Tian, "Molecular optical simulation environment (MOSE): A platform for the simulation of light propagation in turbid media," PLOS ONE, 8(4), e61304 (2013).

13. H. Shen, G. Wang, "A tetrahedron-based inhomogeneous Monte Carlo optical simulator," Phys. Med. Biol. 55(4), 947-962 (2010).

14. Q. Fang, "Mesh-based Monte Carlo method using fast ray-tracing in Plucker coordinates," Biomed. Opt. Exp. 1(1), 165-175 (2010).

15. T. J. Pfefer, J. Barton, E. Chan, M. Ducros, "A three-dimensional modular adaptable grid numerical model for light propagation during laser irradiation of skin tissue," IEEE J. Sel. Top. Quant. 2(4), 934-942 (1996).

16. D. Li, B. Chen, W. Ran et al., "Selection of voxel size and photon number in voxel based Monte Carlo method: Criteria and applications," J. Biomed. Opt. 20(9), 095014 (2015).

17. Y. Zhang, B. Chen, D. Li, G. Wang, "Efficient and accurate simulation of light propagation in biotissues using the three dimensional geometric Monte Carlo method," Numer. Heat Trans. A, Appl. 68(8), 827846 (2015).

18. H. Jia, B. Chen, D. Li, Y. Zhang, Boundary discretization in the numerical simulation of light propagation in skin tissue: Problem and strategy, J. Biomed. Opt. 20(2), 025007 (2015).

19. T. Li, Y. Zhao, Y. Sun, K. Li, Effects of wavelength, beam type and size on cerebral low-level laser therapy by a Monte Carlo study on visible Chinese human, J. Innov. Opt. Heal. Sci. 8(1), 1540002 (2015).

20. Y. Liu, H. Wang, Y. Liu, W. Li, Z. Qian, Monte Carlo and phantom study in the brain edema models, J. Innov. Opt. Heal. Sci. 10(3), 1650050 (2017).

21. K. Zhou, J. Tian, Q. Zhang, X. Meng, K. Yang, Q. Ren, Simulation and quantitative analysis of fluorescence intensity distribution based on the Monte Carlo method, J. Innov. Opt. Heal. Sci. 8(6), 1550038 (2015).
22. T. Li, Y. Li, Y. Sun, M. Duan, L. Peng, Effect of head model on Monte Carlo modeling of spatial sensitivity distribution for functional near-infrared spectroscopy, J. Innov. Opt. Heal. Sci. 8(5), 1550024 (2015).

23. E. Alerstam, T. Svensson, S. Andersson-Engel, "Parallel computing with graphics processing units for high-speed Monte Carlo simulation of photon migration," J. Biomed. Opt. 13(6), 060504 (2008).

24. H. Shen, G. Wang, "A study on tetrahedron-based inhomogeneous Monte Carlo optical simulation," Biomed. Opt. Express 2(1), 44-57 (2011).

25. Q. Fang, D. Boas, Tetrahedral mesh generation from volumetric binary and grayscale images, 2009 IEEE Int. Symp. Biomedical Imaging: from Nano to Macro, Vols. 1 and 2, pp. 1142-1145, Boston, MA (2009).

26. J. Chen, Q. Fang, X. Intes, "Mesh-based Monte Carlo method in time-domain widefield fluorescence molecular tomography," J. Biomed. Opt. 17(10), 106009 (2012).

27. R. Yao, X. Intes, Q. Fang, "Generalized mesh-based Monte Carlo for wide-field illumination and detection via mesh retessellation," Biomed. Opt. Express, 7(1), 171-184 (2016).

28. B. Dogdas, D. Stout, A. F. Chatziioannou, R. M. Leahy, "Digimouse: A 3D whole body mouse atlas from CT and cryosection data," Phys. Med. Biol. 52(3), 577-587 (2007).

29. G. Alexandrakis, F. R. Rannou, A. F. Chatziioannou, "Tomographic bioluminescence imaging by use of a combined optical-PET (OPET) system: A computer simulation feasibility study," Phys. Med. Biol. 50(17), 4225-4241 (2005).

30. E. Alerstam, W. Lo, T. Han, J. Rose, S. AnderssonEngels, L. Lilge, "Next-generation acceleration and code optimization for light transport in turbid media using GPUs," Biomed. Opt. Express, 1(2), 658-675 (2010).

31. Q. Fang, D. Boas, "Monte Carlo simulation of photon migration in $3 \mathrm{D}$ turbid media accelerated by graphics processing units," Opt. Express, 17(22), 20178-20190 (2009). 